\title{
Refining the Role of Autophagy in Hypertrophic Cardiomyopathy
}

\author{
Shuyi Wang and Jun Ren* \\ University of Wyoming College of Health Sciences, USA
}

Submission: December 30, 2017; Published: April 20, 2018

*Corresponding author: Jun Ren, University of Wyoming College of Health Sciences, Laramie, USA, Tel: 1-307-766-6131; Fax: 1-307-766-2954; Email: jren@uwyo.edu

\begin{abstract}
Hypertrophic cardiomyopathy is perhaps the most prevalent form of inherited cardiomyopathy as a result of mutations in genes encoding components of the cardiac sarcomere. Although diagnosis of hypertrophic cardiomyopathy is relatively straightforward, its clinical management has been challenging due to lack of knowledge with regards to the precise pathogenesis of hypertrophic cardiomyopathy. A large body of evidence has recently suggested a pivotal role for impaired protein quality control machinery in inherited cardiomyopathies including hypertrophic cardiomyopathy. Here we will review recent findings on the role of autophagy in the etiology of hypertrophic cardiomyopathy and discuss therapeutic options available to restore autophagy-lysosomal function in an effort to benefit cardiac homeostasis in hypertrophic cardiomyopathy.
\end{abstract}

Keywords: Autophagy; Hypertrophic cardiomyopathy; Cardiac function.

\section{Mini Review}

Cardiomyopathies typically refer to a heterogeneous group of diseases of myocardium accompanied with overt mechanical and electrical abnormalities which result in inappropriate ventricular hypertrophy or dilation [1]. Based on the anatomy and physiology, cardiomyopathy can be classified into five major categories namely dilated, hypertrophic, restrictive, arrhythmogenic right ventricular, and unclassified cardiomyopathies [1-3]. Although there are many forms of cardiomyopathies that develop in a given primary causative disease setting such as diabetic, alcoholic, doxorubicin and septic cardiomyopathies, hypertrophic (HCM) and dilated (DCM) cardiomyopathies are considered the two most prevalent familial forms of cardiomyopathies due to mutations in essential genes encoding proteins of sarcomere, cytoskeleton, sarcoplasmic reticulum, T-tubules and others essential cardiac elements [2-4]. Hypertrophic cardiomyopathy is well known as a monogenetic disease of myocardium with a prevalence of $0.2 \%$ of general population. Hypertrophic cardiomyopathy is a main reason for heart failure and sudden cardiac death with a primary clinical manifestation of cardiac hypertrophy, interstitial fibrosis and cardiomyocyte disarray. Diagnosis of cardiomyopathies is relatively straight forward although early detection of risk populations and clinical treatment remain challenging. In particular, nearly half of sudden cardiac death patients in their early life are afflicted with cardiomyopathies. Although gene mutations in sarcomeric proteins are considered the main causes for hypertrophic cardiomyopathy, the molecular mechanisms that drive the appearance of hypertrophic phenotypes remain elusive. Recent evidence has revealed a number of contributing factors including pathological changes in cardiac sarcomeric structure, ion channels and protein quality control in the etiology of hypertrophic cardiomyopathy [3].

Autophagy is a critical protein quality control process responsible for self-digestion of long-lived or damaged cellular components or organelles. The level of autophagy is usually low under physiological condition and may be induced upon stress stimuli such as starvation, inflammation and hypoxia [5]. Up-to-date, three types of autophagy are identified including macroautophagy, microautophagy and chaperone-mediated autophagy, with macroautophagy being the predominant form. Given that autophagy is a highly conserved dynamic process, it may be defined by several major steps including initiation, elongation, autophagosome formation, autolysosomal formation and finally lysosomal degradation [6]. Disruption of autophagy at any given step can lead to compromised autophagy function. Ample of clinical and experimental data have confirmed an essential role for autophagy in the maintenance of cellular homeostasis in the heart and disruption of which leads to cardiac dysfunction and potentially cardiomyopathy. For example, cardiomyopathy in type 1 diabetes is often presented with impaired autophagosome formation, while diabetic cardiomyopathy in type 2 diabetes usually displays disturbed autophagosome clearance or 'autophagy flux"[7]. Due to variation in etiology, and pathophysiological mechanisms, the 
role of autophagy in different forms of cardiomyopathies is distinct. Here we will briefly summarize limited data available on the role of autophagy in hypertrophic cardiomyopathy.

Changes in autophagy have been noted in hypertrophic cardiomyopathy (HCM) caused by Danon disease, Vici syndrome, and LEOPARD syndrome. In particular, autophagy flux was found to be suppressed in Danon disease, a genetic disorder with marked hypertrophic cardiomyopathy, likely as a result of deficiency in lysosome-associated membrane protein 2 (LAMP2)[8]. LAMP-2 locates on lysosomal membrane and governs the fusion of autophagosome with lysosome. With LAMP-2 insufficiency in Danon disease, autophagosomes are unable to fuse with the lysosomes to initiate the lysosomal degradation process, resulting in large amount of vacuoles accumulated [9]. In Vici syndrome with prominent global developmental delay, skin hypopigmentation, nascent cataracts, microcephaly and hypertrophic cardiomyopathy, recessive mutation is evident in EPG5 (c.5870-1G>A) encoding a key autophagy regulator for autophagosome-lysosome fusion [10]. The condition presents usually early in life. In a recent study, autophagy was evaluated in hypertrophic cardiomyopathy patients carrying mutation of MYBPC3, encodes cardiac myosin-binding protein $\mathrm{C}$ (Mybpc3) and in a Mybpc3-targeted knock in hypertrophic cardiomyopathy mouse model. LC3-II protein levels were found elevated in septal myectomies from hypertrophic cardiomyopathy patients compared with non failing controls and in 60-week-old knock in mice compared with wild-type mouse hearts. Interestingly, autophagic flux was found to be blocked along with accumulation of residual bodies and glycogen. These authors identified that elevated Akt-mTORC1 (mammalian target of rapamycin complex 1) signaling was likely responsible for the blunt autophagy flux in mice with Mybpc3-targeted knockin hypertrophic cardiomyopathy, the effect of which was partially rescued by autophagy induction using rapamycin or caloric restriction[11]. Along the same line of Akt-MTOR overaction, recent findings from our group noted overt hypertrophic cardiomyopathy in mice with phosphatase and tens in homolog deleted from chromosome 10 (PTEN) knock out. Cardiomyocyte-specific PTEN knockout displayed the phenotype of established hypertrophic cardiomyopathy (HCM) as evidenced by unfavorable geometric, functional, and histological changes. Our data also revealed higher cardiac mTOR and suppressed cardiac autophagy. Treatment with autophagy inducer rapamycin reversed autophagy and hypertrophic cardiomyopathy in PTEN knockout mice [12]. These findings from patients carrying MYBPC3 mutations, and mice with Mybpc3-targeted knock in or PTEN deletion clearly suggested a role for defective autophagy (or autophagy flux) in the onset and development of hypertrophic cardiomyopathy, possibly through a mTOR hyperactivation-dependent mechanism.

Although data from our lab and others indicated the utility of autophagy induction in the therapy of experimental hypertrophic cardiomyopathy [11,12], there is still no drug therapy available for hypertrophic cardiomyopathy with heart transplantation being the only possible option. Danon disease is an X chromosome linked defect, and not surprisingly, LAMP2 may serve as the major drug target. Application of DNA methylation inhibitor to reactivate the randomly silenced LAMP2 gene has shown beneficial result in Danon disease, although such effect is limited to a subtype of female patients carrying a wide type allele which is randomly silenced [13]. No information is available with regards to the role of autophagy in DNA methylation inhibitionoffered benefit here.

Drug development targeting autophagy remains challenging due to a number of practical issues. First, a series of steps are involved in autophagy and distinct defects may exist in different clinical situations (such as defect in early phase of autophagosome formation versus late autophagolysosome fusion), a drug that targets autophagy may produce drastically different effect in the treatment of cardiomyopathy, even though the main goal is the same in restoration of autophagy back to normal levels. Several pharmacological agents including bafilomycin, 3-MA, AICAR, and rapamycin are currently used for autophagy regulation, yet understanding of the complex steps and proteins involved still remains limited. Second, autophagy regulators may have offtarget effect. An ideal agent should modulate target proteins and signaling pathways without eliciting any effects on other proteins. This may contribute to unexpected side effect such as application of autophagy regulators in cancer therapy. At this time, limited agents are available with high specificity. Third, organ specificity may be an issue for pharmacotherapy targeting autophagy. Systemic delivery such as oral administration and IV infusion results in global distribution of the agent, which may or may not affect levels of autophagy elsewhere, especially in healthy organs or tissues. Liver toxicity, kidney toxicity may develop for therapeutic doses in the heart. It is quite challenging for targeted local delivery as the invasive nature may generate issues such as trauma, hemorrhage and inflammation.

Despite the challenges and obstacles in the drug development targeting autophagy, correction of autophagy derangement remains a promising avenue in the management of hypertrophic cardiomyopathy. Several aspects can be considered to overcome the aforementioned obstacles in autophagy drug therapy, including how the agent can generate its effect on cardiomyocytes. A number of approaches may be considered in the development of small molecule autophagy regulatory agents. In order to exhibit its local effect, an agent should be hydrophobic to allow diffusion across cell membrane into the intracellular space. Another option is to seek an agent with a natural receptor on the cell membrane where signal transduction can be achieved using the receptor-driven cell signaling regulation. Yet tissue/ organ specificity remains a practical issue for the hunt for selective cardiac drugs for the regulation of autophagy in the management of hypertrophic cardiomyopathy. 


\section{References}

1. Asakura M, Kitakaze M (2014) Classification of cardiomyopathy. Masui 63: 5-15.

2. Kalisz K, Rajiah P (2017) Computed tomography of cardiomyopathies. Cardiovasc Diagn Ther: 539-556.

3. McKenna WJ, Maron BJ, Thiene G (2017) Classification, Epidemiology, and Global Burden of Cardiomyopathies, Circulation research 121: 722-730.

4. Liew AC, Vassiliou VS, Cooper R, Raphael CE (2017) Hypertrophic Cardiomyopathy-Past, Present and Future. Journal of clinical medicine: 6 .

5. Nishida K, Kyoi S, Yamaguchi O, Sadoshima J, Otsu K, et al. (2009) The role of autophagy in the heart, Cell Death Differ 16: 31-38.

6. Gottlieb RA, Mentzer RM (2013) Autophagy: an affair of the heart. Heart Fail Rev 18: 575-584.

7. Kubli DA, Gustafsson AB (2015) Unbreak my heart: targeting mitochondrial autophagy in diabetic cardiomyopathy. Antioxid Redox Signal 22: 1527-1544.

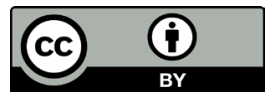

This work is licensed under Creative

Commons Attribution 4.0 License

DOI: 10.19080/IJCSMB.2018.04.555637
8. Nishino I, Fu J, Tanji K, Yamada T, Shimojo S, et al. (2000) Primary LAMP-2 deficiency causes X-linked vacuolar cardiomyopathy and myopathy (Danon disease). Nature 406: 906-910.

9. Rowland TJ, Sweet ME, Mestroni L, Taylor MR (2016) Danon disease - dysregulation of autophagy in a multisystem disorder with cardiomyopathy. J Cell Sci 129: 2135-2143.

10. Touraine R, Laquerriere A, Petcu CA, Marguet F, Byrne S, et al. (2017) Autopsy findings in EPG5-related Vici syndrome with antenatal onset. American journal of medical genetics Part A 173: 2522-2527.

11. Singh SR, Zech ATL, Geertz B, Reischmann-Dusener S, Osinska H, et al. (2017) Activation of Autophagy Ameliorates Cardiomyopathy in Mybpc3-Targeted Knockin Mice. Circ Heart Fail 10.

12. Xu X, Roe ND, Weiser-Evans MC, Ren J (2014) Inhibition of mammalian target of rapamycin with rapamycin reverses hypertrophic cardiomyopathy in mice with cardio myocyte-specific knockout of PTEN. Hypertension 63: 729-739.

13. Ng KM, Mok PY, Butler AW, Ho JC, Choi SW, et al. (2016) Amelioration of X-Linked Related Autophagy Failure in Danon Disease With DNA Methylation Inhibitor, Circulation 134: 1373-1389.

\section{Your next submission with Juniper Publishers will reach you the below assets}

- Quality Editorial service

- Swift Peer Review

- Reprints availability

- E-prints Service

- Manuscript Podcast for convenient understanding

- Global attainment for your research

- Manuscript accessibility in different formats ( Pdf, E-pub, Full Text, Audio)

- Unceasing customer service

Track the below URL for one-step submission https://juniperpublishers.com/online-submission.php 